\title{
Communications
}

\section{Examen de spécialiste \\ Examen de spécialiste en vue de l'obtention du titre de spécialiste en cardiologie}

Examen écrit:

Date: Mercredi, 28 octobre 2015,

Heure: 8:45-12:00

Lieu: Hôpital de l'Ile Berne, Pathologisches Institut, Hörsaal Langhans, Eingang 43a

Examen oral:

Date: Jeudi, 29 octobre 2015

Lieu: Hôpital Universitaire, Zurich (en allemand), Hôpitaux Universitaires de Genève, Genève (en français)

\section{Délai d'inscription: 31 août 2015}

Vous trouverez de plus amples informations sur le site web de l'ISFM www.siwf.ch $\rightarrow$ Domaines spécialisés $\rightarrow$ Titres de spécialiste et formations approfondies (formation postgraduée) $\rightarrow$ cardiologie

Examen Attestation de formation complémentaire en médecine d'urgence hospitalière SSMUS 2015

Examen oral (français et allemand)

Date: 4 décembre 2015

Lieu: Berne, UniS

Examen pratique (français et allemand)

Date: 11 décembre 2015

Lieu: Lausanne, CHUV

\section{Délai d'inscription: 15 août 2014}

Coûts: 600 CHF pour les membres SSMUS / 1000 CHF pour les non-membres

Vous trouvez des informations supplémentaires sur le site de la SSMUS www.sgnor.ch $\rightarrow$ AFC's $\rightarrow$ AFC médecine d'urgence hospitalière SSMUS ou vous les recevez auprès du secrétariat général: sekretariat[at]sgnor.ch

\section{Korrigendum}

Richtigstellung der Eidgenössischen Kommission für Lärmbekämpfung EKLB zum Artikel «Gesundheitsschäden bei Fluglärm» in Ausgabe 8/2015 [1] Im obengenannten Beitrag wird auf Seite 256 behauptet, dass die «Eidgenössische Lärmkommission" (Eidgenössische Kommission für Lärmbekämpfung EKLB), einstimmig entschieden hätte, dass die Fluglärm-Grenzwerte zu hoch angesetzt seien. Dem widersprechen wir:

- Falsch ist, dass die EKLB einstimmig beschlossen hätte, die Fluglärm-Grenzwerte seien $z u$ hoch angesetzt. Es wurde in der Kommission über die Höhe der FluglärmGrenzwerte weder eine Abstimmung durchgeführt, noch hat die Kommission jemals behauptet, die Fluglärm-Grenzwerte (oder Lärm-Grenzwerte allgemein) seien zu hoch.

- Richtig ist, dass die EKLB eine Überprüfung (und ggf. Neufestsetzung) der Grenzwerte in der Lärmschutz-Verordnung für nötig erachtet und dazu ein Forschungskonzept zur Überprüfung der Grenzwerte vorgelegt hat [2]. Dabei lässt es die Kommission grundsätzlich offen, ob Grenzwerte auf jeden Fall herabgesetzt werden müssen oder ggf. auch erhöht werden können.

Prof. Dr. Anne-Christine Favre, Präsidentin der Eidgenössischen Kommission für Lärmbekämpfung EKLB

1 Göschke H. Gesundheitsschäden bei Fluglärm. Schweiz Ärztezeitung. 2015;96(8):265-6.

2 www.eklb.admin.ch/deldokumentationlberichte/ index.html

\section{Ergänzender Hinweis des Autors}

Schon vor Eingang Richtigstellung der Eidg. Kommission für Lärmbekämpfung (EKLB) hatte ich die Ärztezeitung um ein Korrigendum gebeten und meine Formulierung einer "einstimmigen Entscheidung» der EKLB bedauert. Sie war Folge einer missverständlichen Information.

Unbestritten ist jedoch aufgrund der im Artikel zitierten Studien, dass gravierende Gesundheitsschäden, insbesondere Herzinfarkte, vermehrt auftreten bei Fluglärmpegeln weit unterhalb unserer gesetzlichen Lärm-Grenzwerte. Eine neue Meta-Analyse [1] hat dies bestätigt.

1 Vienneau D et al. The relationship between transportation noise exposure and ischemic heart disease: A meta-analysis. Environmental Research. 2015;138:372-80.

PD Dr. med. Hans Göschke, Vorstand Ärztinnen und Ärzte für Umweltschutz, Sektion Basel 